\title{
Impact of Histopathological Examination of Appendix in Context to Clinical Management of Patients
}

\author{
Mandakini M Patel and Rhuta J Shah* \\ Dept. of Pathology, Govt. Medical College, Surat, India
}

\section{ABSTRACT}

Background: Acute appendicitis is one of the common conditions requiring emergency surgery. In routine practice , appendix is sent for histolopathological examination only when the operative findings are inconclusive. In view of this trend in clinical practice this study was carried out to assess the value of routine histopathological examination of surgically resected appendices, to review spectrum of histopathological diagnosis of appendectomy done at our institute over a 22 months period, some of which would further have effect on patient management and prognosis.

Methods: A retrospective study was done including appendicectomy specimens received at histopathology section of Department of Pathology at Tertiary care centre in South Gujarat during January 2015 till October 2016.

Result: Total 400 cases were reviewed. Out of these, clinically suspected appendicitis was found in 365 (91.3\%) cases including spectrum of appendicitis (acute, subacute, chronic, ulcerative, suppurative, necrotizing, gangrenous, acute with periappendicits, acute on chronic). Unusual unexpected findings were found in 20 (5\%) cases[tuberculosis, amoebiasis, faecolith, congestion with sickle RBCs, mucocele and neoplastic lesions including carcinoid, adenocarcinoma, mucinous cystadenoma (low grade appendiceal mucinous neoplasm)].

Conclusion: Though majority of cases had the usual features, 20 of these 400 specimen (5\%) had an impact on patient management or outcome. They were not suspected on macroscopic examination at the time of surgery and would have been missed had the specimens not been examined microscopically. Intraoperative diagnosis of surgeon is therefore unreliable in detecting abnormalities of appendix. This study supports the sending of all appendicectomy specimens for routine histopathological examination.

\section{Keywords: Appendicitis, Unusual Findings, Value}

\section{Introduction}

Acute appendicitis is one of the common conditions requiring emergency surgery. ${ }^{[1]}$ The practice of sending all appendix specimens for routine histopathological examination depends on the concerned clinician and is variable. Matthyssens et al are against this policy and suggest that appendices should be sent for examination only if there is an obvious macroscopic abnormality at surgery ${ }^{[2,3]}$. They justify their opinion by the rarity of aberrant findings, together with the significant costs of specimen processing. However, a number of other papers have found such aberrant incidental findings to be more common, and suggest that failure to histopathologically examine all appendices would lead to many significant pathologies being missed and cause an impact on patient management ${ }^{[2,4-6]}$. There is no authoritative data or previous studies which deal with this issue in India. Hence, a query still remains whether this policy should be adhered to in poor resource countries like India also. Keeping in mind the above facts, we undertook this study to shed some light on this topic by evaluating the current scenario concerning this issue in India.

Histopathological examination still remains the gold standard method for the confirmation of the appendicitis. Not only the pathologic diagnosis of acute inflammation, at times unusual findings such as incidental tumours noted in the appendix highlights the importance of the pathologic analyses of every single resected appendix.

This study aims to determine the various histologic diagnoses of all surgically removed appendices and to find out the age and sex related incidence of appendicitis, rate of negative appendicectomies and unusual findings which would have effect on patient management and care ${ }^{[1]}$

\section{Aims \& Objectives}

(1) To study spectrum of histopathological lesions in appendicectomy specimens. (2) To analyze the proportion of various lesions, age and sex distribution in resected specimens of appendix. (3) To find out proportion of unusual findings in appendicectomy specimen, some of which would have effect on patient management and prognosis. 


\section{Materials and Methods}

A retrospective study was done including appendicectomy specimens received at histopathology section of Department of Pathology at Tertiary Care Center in South Gujarat during 22 months period from January 2015 till October 2016. Total 400 appendicectomy specimens were included in study.

Inclusion Criteria: All emergency appendectomies and interval appendectomies performed on clinically suspected appendicitis were included.

Exclusion criteria: Incidental appendectomies which were performed during other abdominal or pelvic surgeries were excluded.

Relevant clinical data, intraoperative findings and gross findings were noted. Appendicectomy specimens are prepared according to a hospital-defined protocol, involving immediate fixing in formalin prior to transport to the pathology laboratory. Specimens are sectioned at the tip, body and base, processed, slides stained with hematoxylin-eosin and were examined by a consultant or senior pathologist. Data were analysed by Ms Excel and SPSS software. Negative appendectomy was defined as one which is performed for a clinical diagnosis of acute appendicitis but in which the appendix is found to be normal on histopathological examination.

The analysis focused on the confirmation of acute appendicitis, incidental unexpected incidental findings other than inflammation, whether these abnormalities were suspected on gross examination at the time of surgery, and the effect on patient management and prognosis. ${ }^{[7]}$

\section{Result}

Total of 400 specimens of appendix were received in the histopathology department during the period of 22 months from January 2015 to October 2016. There were 284 (71\%) males and $116(29 \%)$ females among 400 cases of appendicitis with the male: female ratio of $2.4: 1$. Overall, a greater number of appendectomies (71\%) were performed in males than in females $(29 \%)$.
The peak age incidence of appendicitis was found in the age group of 21 to 30 years. More than $80 \%$ cases of appendicitis occurred below the age of 40 years. The youngest patient was two years old and the oldest was seventy-five years of age.

Many patients presented with multiple and overlapping clinical symptoms. The most common symptom was pain in abdomen seen in 395(98.5\%) followed by fever $225(56.2 \%)$ and vomiting $118(29.6 \%)$. Patients presenting with intestinal obstruction were $3(0.75 \%)$ patients and perforation were $9(2.25 \%)$.

As shown in table 1, out of total 400 cases reviewed ,clinically suspected appendicitis was proven histologically in $365(91.3 \%)$ cases including spectrum of appendicitis (acute, subacute, chronic, ulcerative, suppurative, necrotizing, gangrenous, acute with periappendicits, acute on chronic). Unusual unexpected findings were found in $20(5 \%)$ cases ( tuberculosis, amoebiasis [figure 1], faecolith, congestion with sickle RBCs, mucocele and neoplastic lesions including carcinoid, adenocarcinoma, mucinous cystadenoma(low grade appendiceal mucinous neoplasm)[figure 2]).

As shown in table 2, total $20(5 \%)$ cases out of 400 were found to have aberrant/ unusual findings. These include 4 cases of Tuberculosis, 3 cases showed trophozoite forms of Amoebiasis. Faecolith was found in 3 cases, retention mucocele in 3 cases. One appendix showed congestion with sickle RBCs. Total 6 (1.5\%) out of 400 turned out to be neoplastic which include 4 cases of Mucinous cystadenoma (low grade appendiceal mucinous neoplasm), 1 case of Carcinoid tumour and 1 case of Adenocarcinoma.

Negative appendectomy was defined as one which is performed for a clinical diagnosis of acute appendicitis but in which the appendix is found to be normal on histopathological examination.11 cases out of 400 in our study accounted for negative appendicectomy. This implies that appendicitis was not cause of acute abdomen

Table 1: Histologic findings of appendicectomy specimen.

\begin{tabular}{|l|c|c|}
\hline Diagnosis & No of cases & $\%$ of cases \\
\hline Acute appendicitis & 142 & $35.5 \%$ \\
\hline Acute Ulcerative appendicitis & 131 & $32.7 \%$ \\
\hline Subacute appendicitis & 38 & $9.5 \%$ \\
\hline Acute necrotizing appendicitis & 24 & $6 \%$ \\
\hline Acute appendicitis with periappendicitis & 10 & $2.5 \%$ \\
\hline
\end{tabular}




\begin{tabular}{|c|c|c|}
\hline Diagnosis & No of cases & $\%$ of cases \\
\hline Acute suppurative appendicitis & 9 & $2.2 \%$ \\
\hline Acute on chronic appendicitis & 7 & $1.7 \%$ \\
\hline Gangrenous appendicitis & 5 & $1.2 \%$ \\
\hline Chronic appendicitis & 7 & $1.7 \%$ \\
\hline NO Appendix & 4 & $1 \%$ \\
\hline NAD & 11 & $2.7 \%$ \\
\hline UNUSUAL FINDINGS & 20 & $5 \%$ \\
\hline
\end{tabular}

Table 2: Unusual findings in appendicular specimen.

\begin{tabular}{|c|c|c|}
\hline Unusual findings & No of cases & $\%$ of cases \\
\hline Tuberculosis & 4 & $1 \%$ \\
\hline Amoebiasis & 3 & $0.75 \%$ \\
\hline Congestion with sickle RBC & 1 & $0.25 \%$ \\
\hline Faecolith & 3 & $0.75 \%$ \\
\hline Retention Mucocele & 3 & $0.75 \%$ \\
\hline NEOPLASTIC & 6 & $1.5 \%$ \\
\hline Carcinoid tumour & 1 & \\
\hline Adenocarcinoma & 1 & \\
\hline Mucinous Cystadenoma (Low grade appendiceal mucinous neoplasm) & 4 & \\
\hline
\end{tabular}

Table 3: Comparison of histopathological findings.

\begin{tabular}{|l|c|c|c|c|}
\hline Histopathological findings & Present study & $\begin{array}{c}\text { Divya R et al } \\
(2016, \text { Aligarh, India) }\end{array}$ & $\begin{array}{c}\text { Hanish Chavda(2015, } \\
\text { Bagalkot, Karnataka, India) }\end{array}$ & $\begin{array}{c}\text { Alun Jones(2007, } \\
\text { England) }\end{array}$ \\
\hline Inflammatory lesions & $365(91.3 \%)$ & $300(92.3 \%)$ & $94.6 \%$ & $77 \%$ \\
\hline UNUSUAL FINDINGS & $20(5 \%)$ & $8(2.5 \%)$ & $4.6 \%$ & $3.75 \%$ \\
\hline
\end{tabular}

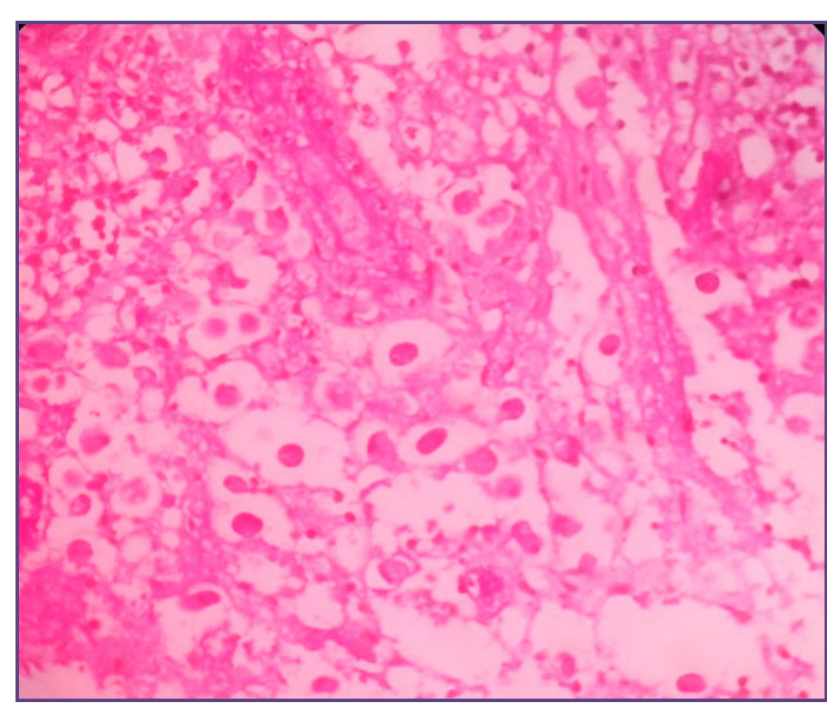

Fig. 1: Trophozoites of Entamoeaba histolytica in appendiceal lumen (H\&E,40x)

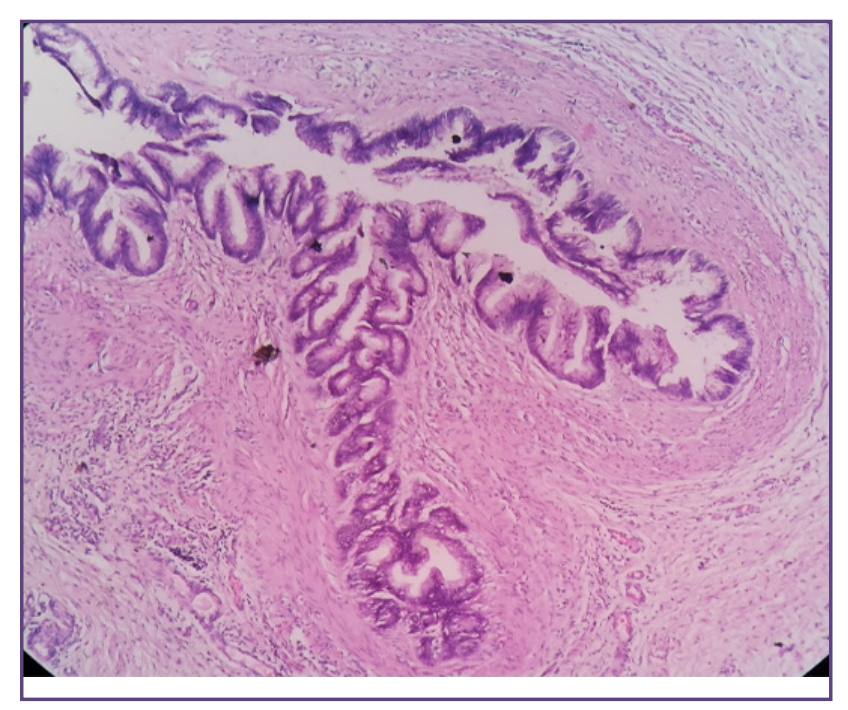

Fig. 2: Mucinous cystadenoma (H\&E , 10x). 


\section{Discussion}

The current study is a two year retrospective study and presents the data on histopathological analyses of 400 appendectomy specimens received in the Department of Pathology at Tertiary Care centre in South Gujarat. The histopathological examination of the appendix serves two purposes. First it allows the diagnosis of acute appendicitis to be confirmed; Second, histopathological examination may disclose additional pathologies that may not be evident intraoperatively which may impact patient management. ${ }^{[8]}$

In our study, age incidence of appendicitis was higher in 20-30 yrs age group. Similar results were seen in study done on 325 cases at Jawaharlal Nehru Medical College by Divya Rabindranath ${ }^{[2]}$ in Aligarh, India. Our result are concordant with study done on 930 cases by Shrestha R et $\mathrm{al}^{[1]}$ in bharatpur, Nepal and by Mohsin-ul-Rasool ${ }^{[8]}$ on 440 cases in Srinagar, India.

In our study, maximum number of patients presenting with complaints of suspected Acute appendicitis were males(71 \%). Similar findings were observed in study done by Divya Rabindranath ${ }^{[2]}$ in Aligarh, India(58.7 \%), Mohsin-ul-Rasool ${ }^{[8]}$ on 440 cases in Srinagar, India(68.2 \%) and by Hanish Chavda ${ }^{[9]}$ in Bagalkot, Karnataka(60.95 $\%)$. In our study most common presenting complaint was abdominal pain.This finding was concordant with study done by Mohsin-ul-Rasool ${ }^{[8]}$ (Srinagar, India) and Edino et al, Nigeria ${ }^{[10]}$.

Negative appendectomy was defined as one which is performed for a clinical diagnosis of acute appendicitis but in which the appendix is found to be normal on histopathological examination.

In our study, rate of negative appendicectomy was 2.7 $\%$. In study done by Mohsin-ul-Rasool ${ }^{[8]}$ (2014, Srinagar, India) reported rate for negative appendicectomy was 5.7 $\%$ and $10.8 \%$ in study done by Shretha R et al ${ }^{[1]}$ (2012, Bhartapur, Nepal). Negative appendicectomy implies that appendicitis was not cause of acute abdomen and other investigations need to be performed if symptoms persist .

In our study, clinically suspected appendicitis appendicitis were histologically correlated in $365(91.3 \%)$ cases as shown in table 3.This findings were concordant with those of Divya R et.al(92.3\%) ${ }^{[2]}$, Hanish Chavda (94.6 \%) [9] and Mohsin-ul-Rasool(77 \%) ${ }^{[8]}$ (Table 3)These include spectrum of inflammatory lesions of appendix , of which acute appendicitis(35.5\%) and acute ulcerative appendicitis(32.7\%), constitute majority of cases. Other in spectrum, constitute minority of proportion including acute suppurative, necrotizing, with periappendicitis, gangrenous and chronic appendicitis.
As shown in table 3, in our study aberrant/unusual findings were found in $20(5 \%)$ of cases. Divya $\mathrm{R}$ et.al ${ }^{[2]}$ found such unusual histological features in $2.5 \%$ of cases. In study done by Hanish Chavda ${ }^{[9]}$, unexpected histological features were found in $4.6 \%$ and $3.75 \%$ in Alun Jones ${ }^{[7]}$. Results are concordant

Another important incidental diagnosis in our study was granulomatous appendicitis which was reported as Tuberculosis due to confirmation on Ziehl-Neelsen staining. Incidence of this rare condition has been reported as $0.14 \%$ to $0.3 \%$ in Western countries and as $1.3 \%$ to $2.3 \%$ in underdeveloped countries ${ }^{[7,11,]}$. It can be caused by various infectious and noninfectious factors. Systemic conditions, such as Crohn's disease and sarcoidosis, may be associated with granulomatous inflammation of the appendix. However, infectious causes like Mycobacterium tuberculosis, Yersinia spp, blastomycosis, Schistosoma spp, Actinomyces spp, Campylobacter spp, and Histoplasma capsulatum form a much more important cause in our country ${ }^{[7,12]}$. Since Tuberculosis is endemic in our country, our case was also suspected to suffer from intestinal tuberculosis. Patient was subsequently investigated for the same and our suspicions were found to be correct(positive Ziehl-Neelsen staining).

The reported incidence of appendicular Tuberculosis varies from $0.1 \%$ to $3.0 \%$ among all appendectomies performed. An accurate diagnosis is usually established only after histopathological examination of a specimen. Some studies report that no further treatment after appendectomy is necessary for primary appendicular disease. In contrast, Jones et al described a case of appendicular Tuberculosis in their study who subsequently underwent right hemicolectomy for treatment. Hence, no consensus has been reached yet about the treatment of appendicular Tuberculosis ${ }^{[7,11]}$.

Acute appendicitis may be the mode of presentation of appendix neoplasms particularly adenocarcinoma ${ }^{[4]}$ as was also seen in our study. One case that was suspected to be acute appendicitis was finally revealed to have adenocarcinoma on histopathological examination. patient with adenocarcinoma should undergo subsequent right hemicolectomy

Carcinoids are the most common tumors of appendix and are typically small, firm, circumscribed yellow brown lesions. An appendiceal carcinoid tumor is found in $0.3 \%$ $2.27 \%$ of patients undergoing an appendectomy $\left.{ }^{[14}\right]$.in our study, we had one case of carcinoid. It has been suggested that carcinoid tumors may present as appendicitis because of luminal obstruction or elevated levels of 5 
hydroxy tryptamine, histamine and kinin as these are all potent mediators of inflammation ${ }^{[13]}$. Characteristics of appendiceal carcinoids predicting aggressive behavior include tumor size, histological subtype, and mesoappendiceal involvement.

Carcinoid tumors are smaller than $1 \mathrm{~cm}$ in $70 \%-95 \%$ of cases [14], including ours. Such small tumors are easily missed on gross examination intraoperatively. The calculated risk of metastasis from tumors $1 \mathrm{~cm}$ or smaller are reported to be nearly zero and therefore may be managed with a simple appendectomy. An increase in metastasis risk of up to $85 \%$ occurs with a tumor double the size or larger. An appendiceal carcinoid tumor larger than 2 $\mathrm{cm}$ should be managed with a formal right hemicolectomy $[14,15]$. Since our particular case was about $1 \mathrm{~cm}$ in size, no further management was needed..

Our study also included three cases of mucocele. A mucocele of the appendix denotes an obstructive dilatation of the appendiceal lumen due to abnormal accumulation of mucus, which may be caused either by a retention cyst, endometriosis, mucosal hyperplasia, cystadenoma, or a cystadenocarcinoma. The incidence of mucocele has been reported to range from $0.2 \%$ to $0.3 \%$ of all appendectomy specimens. We have seen 3 cases of retention mucocele in our study Mucoceles are often asymptomatic and discovered only as incidental findings at appendicectomy, or during laparotomy for another indication or at histological examination of an operative specimen. However, they may also be diagnosed clinically from features of acute appendicitis. Confirmative diagnosis of mucocele and its cause is possible only after histopathology. Appendectomy is the treatment of choice for mucinous cystadenoma, whereas a cystadenocarcinoma requires a right hemicolectomy. Because of the high association of mucinous cystadenoma with colon and ovarian malignancy, follow-up Computed tomography, Ultrasonography, and colonoscopy examinations must be performed during the postoperative period ${ }^{[14]}$.

Hence, our study demonstrated that histopathological examination led to the incidental diagnosis of many important lesions that would have been otherwise missed by the surgeon. These diagnoses led to significant effect on patient management. These included conditions like Low grade Appendiceal Mucinous neoplasm (Mucinous cystadenoma) and mucinous cystadeno carcinoma which have a high risk of association with other neoplasms. Hence, their diagnosis is imperative for adequate patient management. Also, few conditions can be first diagnosed in appendix only, like granulomatous appendicitis (due to tuberculosis or crohn`s disease), amoebiasis, cancers like carcinoid and adenocarcinoma .Thus, such incidental detection can lead to early treatment of these conditions.

It becomes obvious from the above discussion that it is highly beneficial to send all appendectomy specimens for histopathological examination. When we weigh the cost of the procedure against the possible benefits, it becomes clear that the benefit far outweighs the cost in this situation. Early diagnosis and treatment of a lesion would prevent the added costs the patient would have to bear if the diagnosis was late and the disease had spread to other organs.

\section{Conclusion}

Despite of advances in technology and imaging modalities there is dilemma in the clinical diagnosis of acute appendicitis. Histopathological examination still remains the gold standard method for the confirmation of the appendicitis.

The histopathological examination of the appendix serves two purposes. First, it allows the diagnosis of acute appendicitis to be confirmed, especially where this is not evident intra-operatively. Second, histopathological examination may disclose additional pathologies that may not be evident on gross examination intra-operatively but may affect subsequent clinical management of the patient.

\section{Implication of Research}

Abberant/unusual findings discovered at Histopathological analysis were not suspected on macroscopic examination at the time of surgery and had an impact on patient management and outcome. These would have been missed had the specimens not been examined microscopically.

The intraoperative diagnosis of the surgeon is therefore unreliable in detecting abnormalities of the appendix. This study supports the sending of all appendicectomy specimen for routine histopathological examination and meticulous examination of all of those.

\section{Acknowledgements}

We acknowledge support of our departmental head and technical assistance of staff concerned with histopathology processing unit.

\section{Reference}

1. Shrestha R, Ranabhat SR, Tiwari M: Histopathologic analysis of appendectomy specimens. Journal of Pathology of Nepal. 2012:2;215 -219

2. Rabindranath D, Khan AA, Ansari H, Senthil P. Unusual incidental findings of routine histopathological examination of appendectomy specimens- a 2-year retrospective analysis with review of the literature. Int J of Allied Med Sci and Clin Res 2016; 4(1):90-98. 
3. Matthyssens LE, Ziol M, Barrat C, Champault GG: Routine Surgical Pathology in General Surgery. Br J Surg 2006; 93:362-368

4. Chan W, Fu KH: Value of Routine Histopathological examination of appendices in Hong Kong. J Clin Pathol 1987; 40:429-433.

5. Duzgun AP, Moran M, Uzun S, Ozmen M, Ozer VM, Seckin S, Coskun F: Unusual findings in appendicectomy specimens: Evaluation of 2458 cases and review of the literature. Indian J Surg 2004; 66(4):221-226.

6. Nemeth L, Reen DJ, O'Briain D, McDermott M, Pui P: Evidence of an Inflammatory Pathologic Condition in "Normal" Appendices following Emergency Appendectomy. Arch Path Lab Med 2001; 125(6):759-764.

7. Jones AE, Phillips AW, Jarvis JR, Sargen K. The value of routine histopathological examination of appendicectomy specimens, Bio Medical Central surgery 2007;7:17

8. Sharma S, Mahajan D, Mohsin-ul-Rasool, Bashir S, Hafiz A, Wajahat M. Histopathology of Appendicectomy Specimen:
A 5 Year Study. Scholars Journal of Applied Medical Sciences. 2014; 2(1B):176-180

9. Chawda HK, Miskin AT, Dombale VD. Spectrum of histopathological lesion in surgically removed appendix, Journal of Drug Discovery and Therapeutics Available. 2015:3;53-56

10. Edino ST, Mohammed AZ, Ochicha O, Anumah M. Appendicitis in Kano, Nigeria: A 5 year review of pattern, morbidity and mortality, Ann Afr Med 2004;3:38-41..

11. Gaffar AB. Granulomatous diseases and granulomas of the appendix. Int J Surg Pathol2010; 18: 14-2

12. Tucker ON, Healy V, Jeffers M, Keane FB. Granulomatous appendicitis. Surgeon2003;1(5):286-289

13. Cortina R, McCormick J, Kolm P, Perry RR. Management and prognosis of adenocarcinoma of the appendix. Dis Colon Rectum 1995; 38: 848-52

14. Sieren LM, Collins JN, Weireter LJ, Britt RC, Reed SF, Novosel TJ, Britt LD. The incidence of benign and malignant neoplasia presenting as acute appendicitis. Am Surg 2010; 76: $808-811$

*Corresponding author:

Dr. Rhuta J Shah, Navkar, Opp. Deep Kiran Appt. Bhagyoday Soc. Chala, Vapi-396191, dist-Valsad, Gujrat(India)

Phone: +91 09428159081

Email: dr.rhutashah@gmail.com

Date of Submission : 23.04.2017

Date of Acceptance : 09.09.2017

Financial or other Competing Interests: None.
Date of Publication : 19.12.2017 